\title{
Efficient electrocardiogram signal compression algorithm using dual encoding technique
}

\author{
Rawaa Qasim Hamza, Khalida Shaaban Rijab, Mohammed Abdul Redha Hussien \\ Department of Electrical and Electronic Engineering, College of Engineering, University of Technology, Baghdad, Iraq
}

\begin{abstract}
Article Info
Article history:

Received May 27, 2021

Revised Nov 15, 2021

Accepted Dec 24, 2021

\section{Keywords:}

Compression

Discrete wavelet transform

DWT threshold

Electrocardiogram

Huffman encoding

Quantization

Run length encoding

ABSTRACT

In medical practices, the storage space of electrocardiogram (ECG) records is a major concern. These records can contain hours of recording, necessitating a large amount of storage space. This problem is commonly addressed by compressing the ECG signal. The proposed work deal with the ECG signal compression method for ECG signals using discrete wavelet transform (DWT). The DWT appeared as powerful tools to compact signals and shows a signal in another time-frequency representation. It is very appropriate in the elimination \& removal of redundancy. The ECG signals are decomposed using DWT. After that, the coefficients that result from DWT are threshold depending on the energy packing efficiency (EPE) of the signal. The compression is achieved by the quantization and dual encoding techniques (run-length encoding \& Huffman encoding). The dual encoding technique compresses data significantly. The result of the proposed method shows better performance with compression ratios and good quality reconstructed signals. For example, the compression ratio $(\mathrm{CR})=20.6,10.7$ and 11.1 with percent root mean square difference (PRD) $=1 \%, 0.9 \%$ and $1 \%$ for using different DWT (Haar, db2 and FK4) Respectively.
\end{abstract}

This is an open access article under the CC BY-SA license.

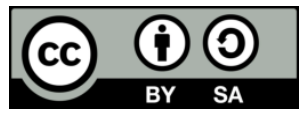

\section{Corresponding Author:}

Rawaa Qasim Hamza

Department of Electrical and Electronic Engineering, College of Engineering, University of Technology

Baghdad, Iraq

Email: 316314@student.uotechnology.edu.iq

\section{INTRODUCTION}

The evaluation of cardiac function is critical for determining heart defects. An electrocardiogram (ECG) is a form of cardiac test used to detect heart problems. It can provide doctors with important medical information. Electrocardiogram (ECG) refers to the measurement of electrical activity in the heart muscles using electrodes attached to the patient's body. The electrophysiological system generates this signal. ECG data that has been compressed needs fewer memory capacity for storage and improves data transfer performance. In the ECG signal, there are peaks and valleys. In ambulatory or continuous cardiovascular disorders, continuous ECG monitoring is needed (CVDs). Continuous ECG monitoring necessitates a large volume of information. The big data necessitates a large storage area,this require a strategy that decreases the data rate thus retaining important medical data during restoration. Highly effective compression methods of low numerical complexity are in high demand in the medical industry. These compression methods may be used in a number of settings, including ambulatory, remote health care, and mobile health care. Recreating the signal is often essential in order to keep the clinically important parameters in the signal. As a consequence, a good compression technique is essential. The three categories of compression strategies are direct, transform-based, and parameter extraction approaches. 
The direct methods depend on explicitly identifying redundancies in the ECG signal. Direct compression approaches include turning point (TP), amplitude zone time epoch coding (AZTEC), and coordinate reduction time encoding scheme (CORTES) [1], [2]. In the transform-based procedure, the original ECG samples are transformed. Compression is then carried out in the transformed domain utilizing methods such as the Fourier transform, wavelet [3], [4]. Transform-based techniques have a higher compression ratio (CR) than direct techniques and are less susceptible to noise in the ECG signal. The ECG signals' properties are maintained by the use of parameter extraction methods. Due to these advantages, the bulk of researchers are concentrating their efforts on transform-based compression techniques. The researcher has experimented with wavelet transforms (WT), fast Fourier transforms (FFT), and discrete cosine transforms (DCT) [5], [6]. The fundamental principle of transformation is to compact the energy of the signal into fewer samples than in the time domain, enabling one to discard tiny transform coefficients (set them to zero). The wavelet transform has a good localization property in the time and frequency domains, which is exactly in line with the transform compression concept [7], [8].

In the literature, many studies have been established to study ECG signal compression as in Ranjeet et al. [9] Introduce a work for compressing an ECG signal based on applying a various transformation kinds like as DWT, FFT, and DCT with Huffman coding algorithm, the simulation results showed a DCT and FFT have a higher compression ratio with $\mathrm{CR}=6.58$ and percent root mean square difference $(\mathrm{PRD})=9.53$ for $\mathrm{DCT}$ and $\mathrm{CR}=5.31$ and $\mathrm{PRD}=9.34$ for FFT whilst a DWT give a parameter with good fidelity with $\mathrm{CR}=3.68$ and $\mathrm{PRD}=8.17$. Chouakri et al. [10] presented a work for compressing an ECG signal by utilizing WT and Huffman coding algorithm, this method planned to transmit an ECG signal through telecommunication medium. The suggested method achieved $\mathrm{CR}=1: 8$ and $\mathrm{PRD}=7 \%$. Abo-Zahhad et al. [11]: presented a hybrid ECG compression technique based on DWT and decreasing the correlation between signal samples and beats by utilizing modified run-length algorithm. The suggested algorithm is tested on records from MIT/BIH database and illustrate the excellent quality of the reconstructed signal with percentage-rootmean square difference less than $1.5 \%$ and compression ratios greater than 20 .

This work introduced an efficient ECG signal compression algorithm based on DWT and dual encoding technique (Huffman encoding and run length encoding). DWT converts ECG signal from the time domain to frequency domain and it compacts energy of the signal to lower-order frequency coefficients. Then, the DWT coefficients are threshold depending on the energy packing efficiency (EPE) of the signal. After that by Using quantization least significant samples are discarded to achieve a higher compression ratio. To encode the signal dual encoding technique has been used which comprised of run-length encoding followed by Huffman encoding to further compresses the signal. To reconstruct the ECG signal, inverse DWT, De-quantizatin, Huffman decoding, and run-length decoding is used. This paper is planned as. Section 2 gives an overview of wavelet transform. Section 3 explains the methodology for ECG signal compression. Section 4 show the effect of noise on ECG signal. Section 5 implement the experimental results of the proposed methods and the discussion. Section 6 present the conclusion of this work.

\section{WAVELET TRANSFORM}

In non-stationary signal processing as well as coding applications such as image encoding and signal, the wavelet transform has been used extensively. The continuous wavelet transformation is known for a signal $\mathrm{x}(\mathrm{t})$ as:

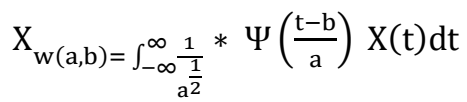

when $\Psi(\mathrm{t})$ denotes the wavelet of analysis (mother wavelet), a time scale is denoted, $\mathrm{b}$ is the time translation and $*$ the complex conjugate is indicated.

The continuous transform wavelet is computed with $\mathrm{x}(\mathrm{t})$ in conjunction with $\left(1 / a^{\frac{1}{2}}\right) \Psi[(\mathrm{t}-\mathrm{b}) / \mathrm{a}]$. As shown in (1), when a and $\mathrm{b}$ are continuous, it is clear that the transformation of the wavelet is quite redundant. A and $\mathrm{B}$ are normally specimens on a time-scale plane on a dyadic grid where $\mathrm{j}$ and $\mathrm{k}$ are $\mathrm{Z}$ and $\mathrm{Z}$ are the integral set, respectively, $a=2^{j}$ and $b=k 2^{j}$, respectively. This is the product of the wavelet sequence [12], [13].

$$
X_{W}(j, k)=\int_{-\infty}^{\infty} 2^{-j / 2}\left(2^{-j} t-k\right) X(t) d t
$$

It is worth noting that $\mathrm{x}(\mathrm{t})$ in the wavelet sequence is a continuous time signal. When $\mathrm{x}(\mathrm{t})$ is a discreet time signal, the transformation of the wavelet lead to become discrete wavelet transform (DWT), which has been written as (3).

$$
X_{w}(j, k)=\sum_{n} 2^{-j / k}\left(2^{-j} n-k\right) X(n)
$$


According to the literature, as seen in Figure 1, discrete wavelet coefficients can be calculated utilizing a filter bank [14]. In Figure 1, it is obvious that, in filter operations, the detail coefficient, d(j,k) and the scale coefficient $\mathrm{c}(\mathrm{j}, \mathrm{k})$ are calculated.

$$
\begin{aligned}
& d_{j, k}=\sum_{n} g(2 k-n) C_{j-1, n} \\
& C_{j, k}=\sum_{n} h(2 k-n) C_{j-1, n}
\end{aligned}
$$

The low-pass filter $\mathrm{h}(\mathrm{n})$ and the high pass filter $\mathrm{g}(\mathrm{n})$ are defined as [14]:

$$
\begin{aligned}
& \mathrm{h}(\mathrm{n})=\frac{1}{2^{1 / 2}} \int \phi\left(\frac{\mathrm{x}}{2}\right) \varphi(\mathrm{x}-\mathrm{n}) \mathrm{dx} \\
& \mathrm{g}(\mathrm{n})=\frac{1}{2^{\frac{1}{2}}} \int \Psi\left(\frac{\mathrm{x}}{2}\right) \varphi(\mathrm{X}-\mathrm{n}) \mathrm{dx}
\end{aligned}
$$

Regarding the mother wavelet and the scaling function are $\Psi(\mathrm{x})$ and $\phi(\mathrm{x})$. where the following criteria must be met by both filters [14]:

$$
(\mathrm{X})=\sum_{\mathrm{n}} \mathrm{g}(\mathrm{n}) \phi(2 \mathrm{x}-\mathrm{n}), \phi(\mathrm{x})=\sum_{\mathrm{n}} \mathrm{h}(\mathrm{n}) \phi(2 \mathrm{x}-\mathrm{n}), \sum_{\mathrm{n}} \mathrm{h}[\mathrm{n}]=2^{\frac{1}{2}}, \sum \mathrm{g}(\mathrm{n})=0, \mathrm{~g}(\mathrm{n})=(-1)^{\mathrm{n}} \mathrm{h}(1-\mathrm{n}) .
$$

Regarding signal $\mathrm{x}(\mathrm{n})$ decomposition, both filters represent decomposition filters. With the same filters, the initial signal may be fully rebuilt.

$$
\left.C_{j-1, k=\sum n}\left[h(2 n-k) C_{j, n}\right]+g(2 n-k) d j, n\right]
$$

The signal is decomposed and reconstructed by the same wavelets, according to (8). For this function, Daubechies [15] proposed orthonormal wavelets with compact support.

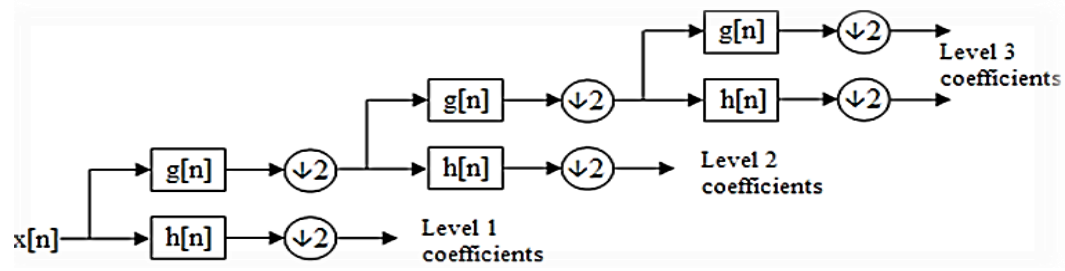

Figure1. DWT Filter bank representation

\section{RESEARCH METHOD}

\subsection{Wavelet decomposition}

We may use DWT at specific periods to remove frequency components from data [16]. During decomposition with a high-pass and low-pass filter, the signal is divided into two sections [17]. For a good quality restored signal with a high level of compression, the mother wavelet should be compactly supported. By the compact support, the discrete wavelet transform is able to work on finite signals in order to distinguish the features of signal in both scale and time [18]. DWT is used with Haar, Daubechies and FK4 wavelets to decompose the ECG signal. Maximum energy is concentrated in the lower approximation sub band for the decomposed wavelet coefficients. The wavelet coefficient amplitudes in a details sub bands (high-frequency wavelet coefficients) in comparison to the subband of approximation small (low-frequency wavelets coefficients) are small. Based on many parameters, the best wavelet filter can be selected. For eg, the optimum wavelet filter has to minimize the error variance reconstructed. the mother wavelets are chosen by depended on the energy conservation property in the wavelet coefficients approximation side, after that the degree of DWT decomposition, which is usually defined by the kind of signal analyzed is selected.

\subsection{Thresholding of the DWT coefficients}

The optimal level is preferred such that the restored signal is as close as possible to the initial. The precision of the signal depends on the determination of the threshold. Benzid et al. [19] a threshold is determined by the energy packing efficiency (EPE) of the signal. 
$\mathrm{EPEi}=\frac{\sum_{\mathrm{n}=1}^{\mathrm{L} 1}(\mathrm{c}(\mathrm{n}))^{2}}{\sum_{\mathrm{n}=1}^{\mathrm{L} 1}(\mathrm{c}(\mathrm{n}))^{2}}$

The thresholding will set a substantial numbers of wavelet coefficients to zeros whose absolute value are lower than a certain threshold level. After thresholding each nonzero coefficient is quantized.

\subsection{Quantization of non-zero threshold coefficients}

The quantizer [20] reduces the amount of bits used to store them by reducing the accuracy of the transformed coefficients. Quantization is a method for transforming a wide range of input values into less output values. During the quantization procedure, some data are lost. As a consequence, the rebuilt signal is distorted, so the quantization level must be chosen precisely at this point. The index for the word code is quantized with 8 bits (256 levels) in these works. The quantized coefficients are subsequently encoded.

\subsection{Coding the wavelet coefficients}

Signal compression aims to represent a limited number of important coefficients, along with big set of zero symbols, in a compact form that can effectively encrypt. After application of the transformation of wavelet to the ECG signal, the amount of zeros and small coefficients are obtained. As small wavelet coefficients with thresholding are substituted by zeros, the amount of zeroes increases. If the wavelets coefficients are store after thresholding, no compression is done, because zero capacity includes memory space as well. The utility of wavelet transforming for ECG compression becomes apparent, as the encoding is used to take advantage of the sequence of zeros. If encoding is added directly to the samples of speech the most compression is not achieved. As the wavelet transform grants structured data with a zero-sequence, encoding ensures high compression afterwards. This requires certain encoding approaches to take benefit of the transformation wavelet for the compression of speech. The zeros are provided by less bit amount when using the encoding. In this study, the ECG signal is encoded using run length encoding (RLE), and Huffman encoding techniques. RLE method is attractive to the ECG compression, as it utilizes data repetition and the compression ratio that obtained from using this method is good [21]. Huffman coding is then employed to maximise compression using a probability dependent encoding methodology. It often uses data repetition. Huffman encoding utilizes a symbol dictionary and encoded signal. In order to evaluate the compressed signal size, both the encoded signal and the dictionary are essential. Strongly Huffman encoding affects on the compression ratio [22], the flow chart of ECG signal compression is shown in Figure 2.

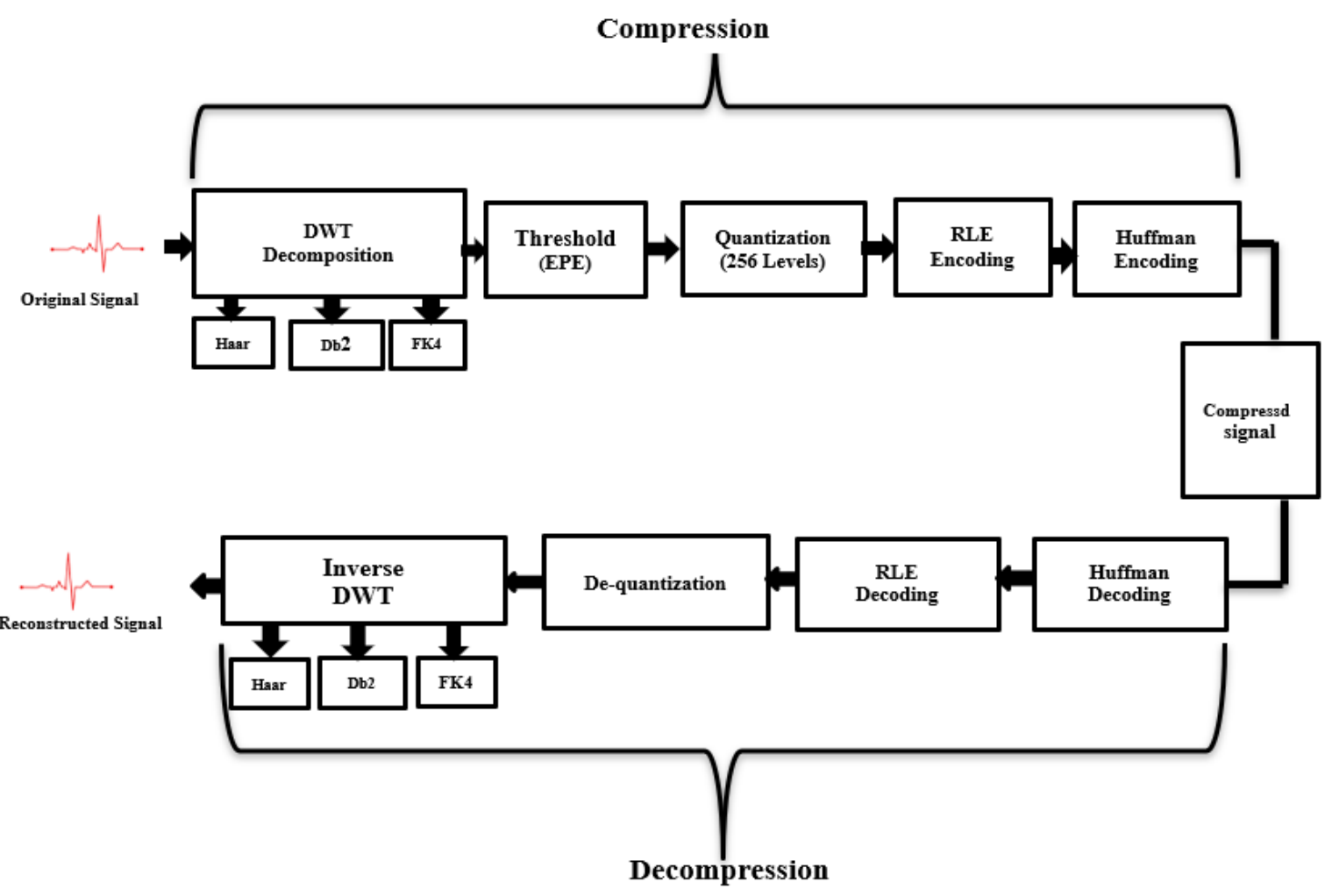

Figure 2. The block diagram of proposed ECG compression method (compression and decompression) 


\section{DE-NOISING BY THRESHOLDING}

The process of threshold plays a significant role in achieving the compression, in addition to removing the noise that affects on a signal. A small coefficient near to zero include a few information and these coefficients affected by noise. In below equation suppose the signal s (k) distorted with noise n:

$$
\operatorname{Sn}(k)=S(k)+n
$$

when applying DWT on the signal that affected by the noise Sn $(\mathrm{k})$, the noise controls on small coefficients of wavelet and the energy of signal is focused into little number of big coefficients [23]. If inverse discrete wavelet transform (IDWT) is used after small coefficients of wavelet are removed via threshold, a rebuilt signal becomes free from the noise. In this work in order to observe the de-noising effect, we add white Gaussian noise to some signals of ECG that taken from MIT/BIH arrhythmia database. the high frequency noises had been simulated using a white Gaussian noise level (WGN) of (5db, 10db, 15db, 20db).

Figure 3 offers the influence of white Gaussian noise on a CR, and we note that the compression ratio is very little affected when adding large amounts of noise, here we can show the system's ability to maintain the compression ratio despite the addition of noise, and the reason is due to the threshold that destroy a large amount of noise. Figure 4 shows the effect of white Gaussian noise (WGN) on percent root mean square difference (prd).

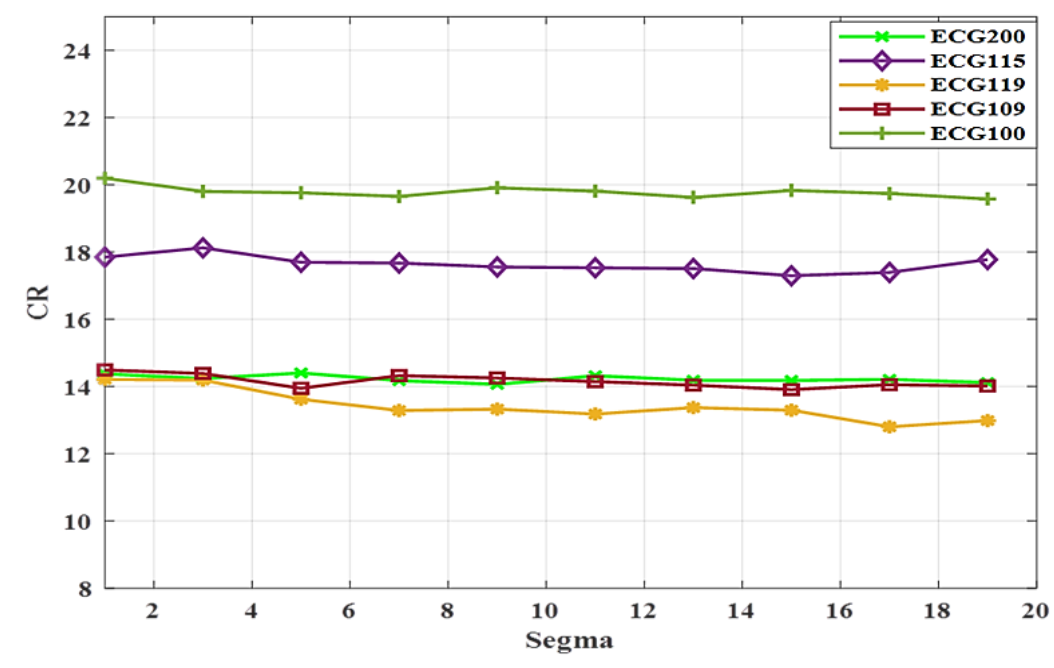

Figure 3. Shows the effect of WGN on the CR

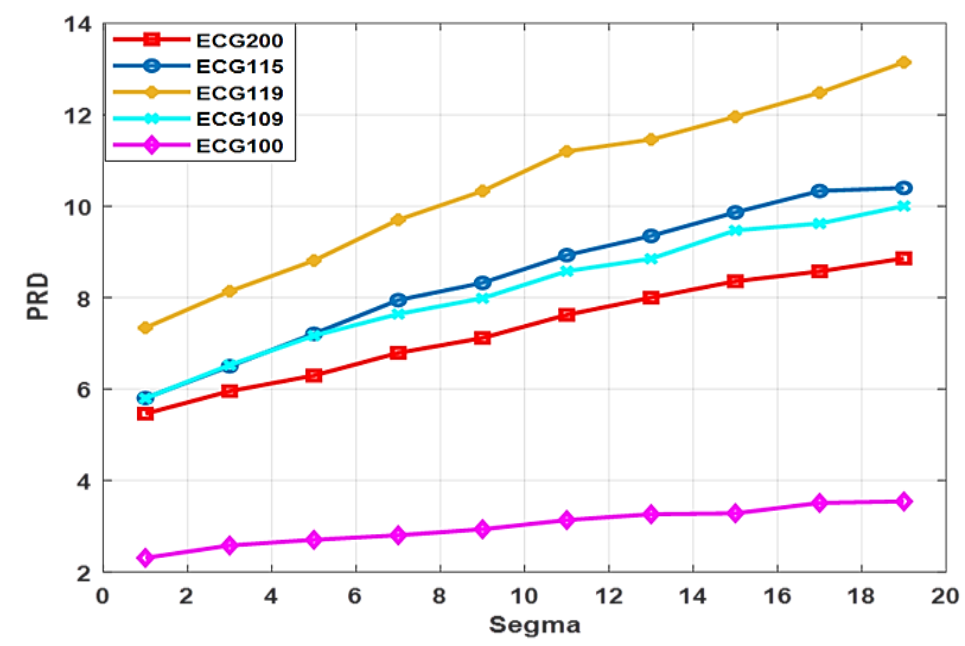

Figure 4. Shows the effect of WGN on the PRD 
We notice when increasing the amount of the added noise to the ECG signal, the amount of prd is increase, but the amount of this rising is small and does not affect on the ECG signal clinical features, a returned signal after the compression is identical to the signal before compression (original signal). Figures 5 and 6 show the result of de-noising when adding (20 db and $15 \mathrm{db}$ ) white Gaussian noise to the signals (119 and signal 115) respectively.
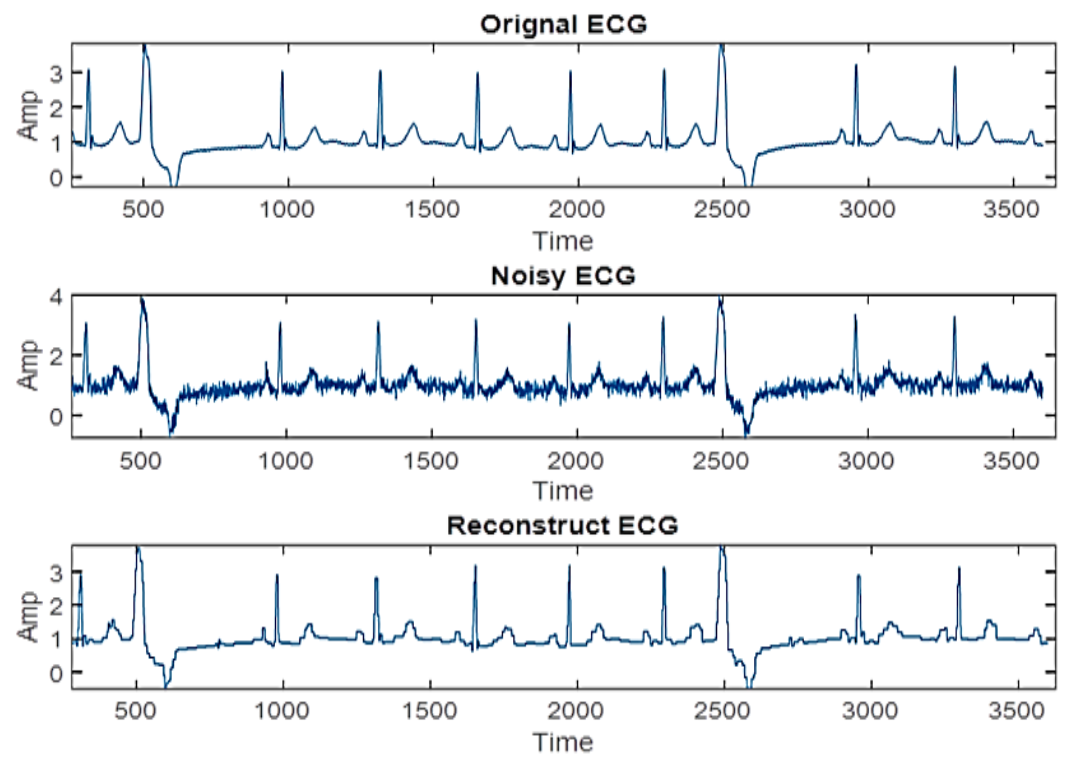

Figure 5. Shows a de-noising result of add $(20 \mathrm{~dB})$ white Gaussian noise to signal (No. 119)
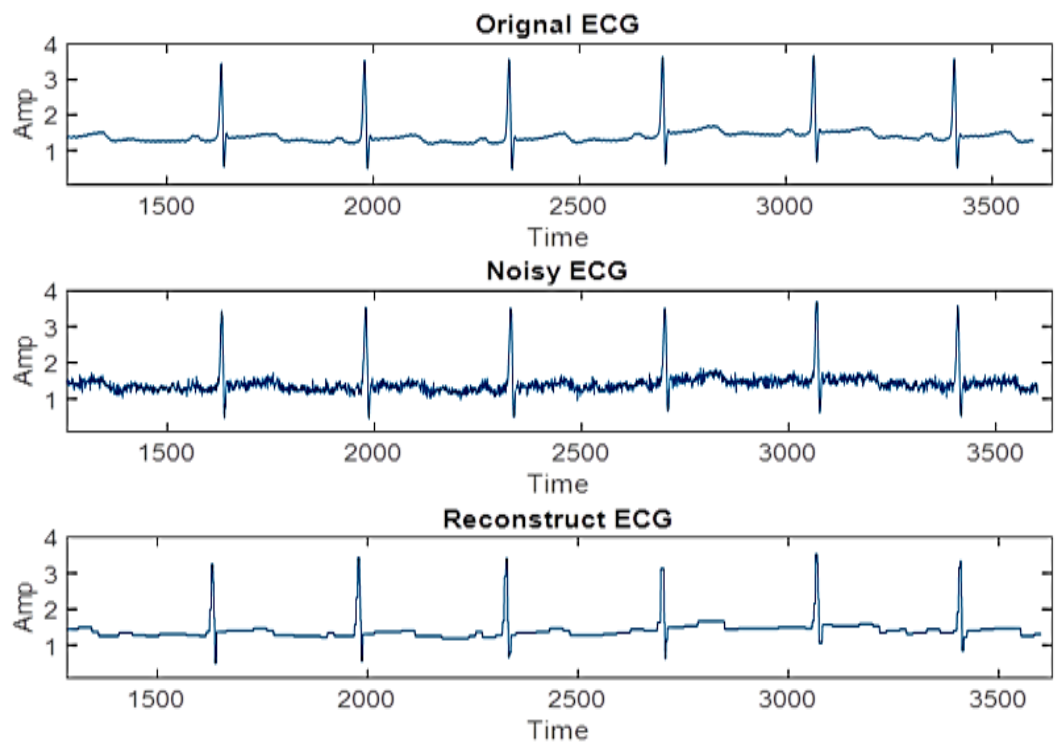

Figure 6. Illustrates a de noising result when add $15 \mathrm{~dB}$ WGN to signal (No. 115)

\section{RESULTS AND DISSCUSSION}

The suggested algorithm was examined on signals from the MIT/BIH database. The characterization of Whole records was (11 bits) resolution and sample frequency $(\mathrm{FS}=360) \mathrm{Hz}$. A set of data consists of record numbers $[100,109,115,119,200]$. The block size for each signal is 3600 samples. The analysis is carried out on Matlab (R2018b) version. Different performance measures were utilized to assess the reliability of rebuilding (CR, PRD, SNR, MSE and RMS). All ECG records have been analysis up to 5 level 
decomposition utilizing different family of wavelets (Haar, $\mathrm{db} 2, \mathrm{fk} 4)$, The 256 quantization levels are used to quantized filter DWT coefficients. From observing the results, we can see that the effectiveness of the suggested algorithm rely on the wavelet that is used and a record of ECG signal being compressed. Table 1 shown the results for the compression of ECG signals by various DWT wavelets and dual coding techniques (Huffman + RLE) encoding technique. From the results in Table 1.

- The best (CR) is obtained when applied "haar" wavelet for whole signals compare with Other DWT types. The $\mathrm{CR}=20.6$ by using DWT (Haar) when the $\mathrm{CR}=10.7$ for DWT $(\mathrm{db} 2)$, and $\mathrm{CR}=11.1 \mathrm{DWT}$ (Fk4) for the same recorder.

- The record (100) obtains the higher CR with 20.6, but the record 119 achieved lower CR=14.16 for the same compression algorithm. That mean the compression ratio change with change the ECG signals.

- The proposed algorithm achieved maximum $\mathrm{PRD}=6 \%$ for the record 119 while the record 100 achieved $\mathrm{PRD}=1 \%$. The results different from one record to another for the same applied algorithm, this thing depend on the record that being compressed it and the type of wavelet that is used.

Figures 7-9 shows the original and reconstructed signals for ECG record $(109,200,100)$ the reconstruct signal is very close to the original signal in all figures.

Table 1. Shown the results for the compression of ECG signals

\begin{tabular}{ccccccc}
\hline \multirow{2}{*}{ Wavelet } & \multirow{2}{*}{ Factor } & 100 & 109 & 115 & 119 & 200 \\
\hline \multirow{2}{*}{ Haar } & CR & 20.6 & 15 & 18.11 & 14.16 & 14.96 \\
& PRD & $1 \%$ & $5 \%$ & $5 \%$ & $6 \%$ & $5 \%$ \\
& SNR & 39.34 & 25.16 & 25.33 & 23.23 & 25.68 \\
& RMS & 0.051 & 0.0839 & 0.0773 & 0.0783 & 0.0933 \\
& MSE & 0.002 & 0.0070 & 0.0060 & 0.0061 & 0.0087 \\
Db2 & CR & 10.7 & 8.41 & 10.31 & 8.16 & 8.76 \\
& PRD & $0.9 \%$ & $3.7 \%$ & $4 \%$ & $4 \%$ & $4 \%$ \\
& SNR & 40.13 & 28.46 & 26.84 & 26.13 & 27.96 \\
& RMS & 0.047 & 0.0574 & 0.0650 & 0.0561 & 0.0718 \\
& MSE & 0.0027 & 0.0033 & 0.0042 & 0.0031 & 0.0052 \\
& CR & 11.1 & 8.03 & 9.5 & 7.6 & 8.1 \\
& PRD & $1 \%$ & $4 \%$ & $4 \%$ & $6.1 \%$ & $4 \%$ \\
& SNR & 39.55 & 26.14 & 26.12 & 24.21 & 27.02 \\
& RMS & 0.050 & 0.075 & 0.070 & 0.069 & 0.079 \\
& MSE & 0.002 & 0.005 & 0.005 & 0.004 & 0.006 \\
\hline
\end{tabular}
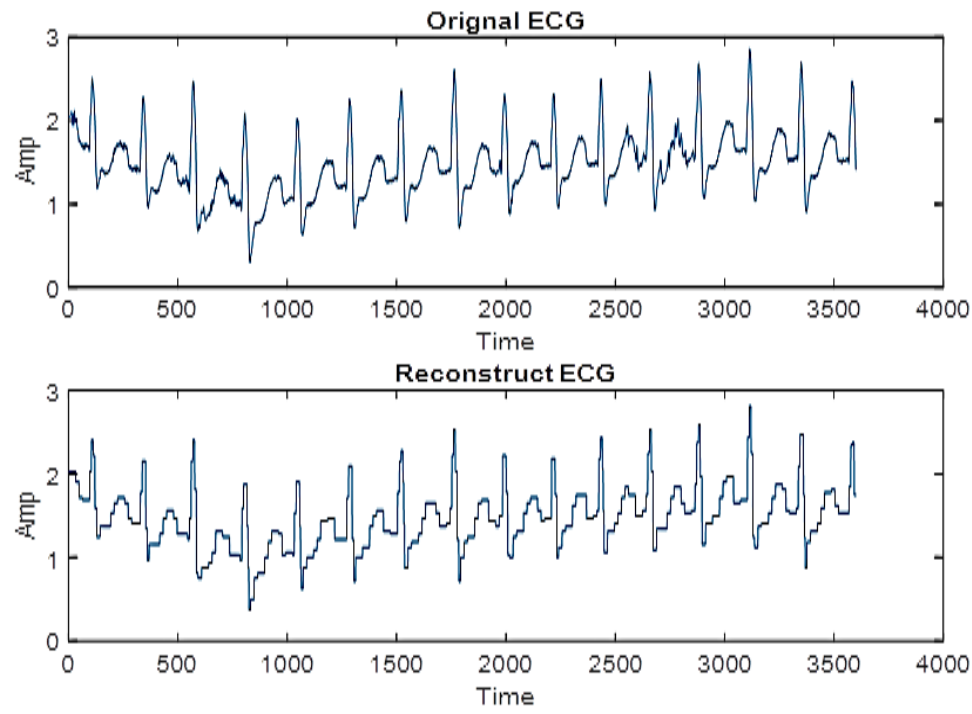

Figure 7. Shows the original and reconstructed signal for ECG record 109 with $C R=15 \& \mathrm{PRD}=5 \%$ we notice the reconstruct signal is very close to the original signal 

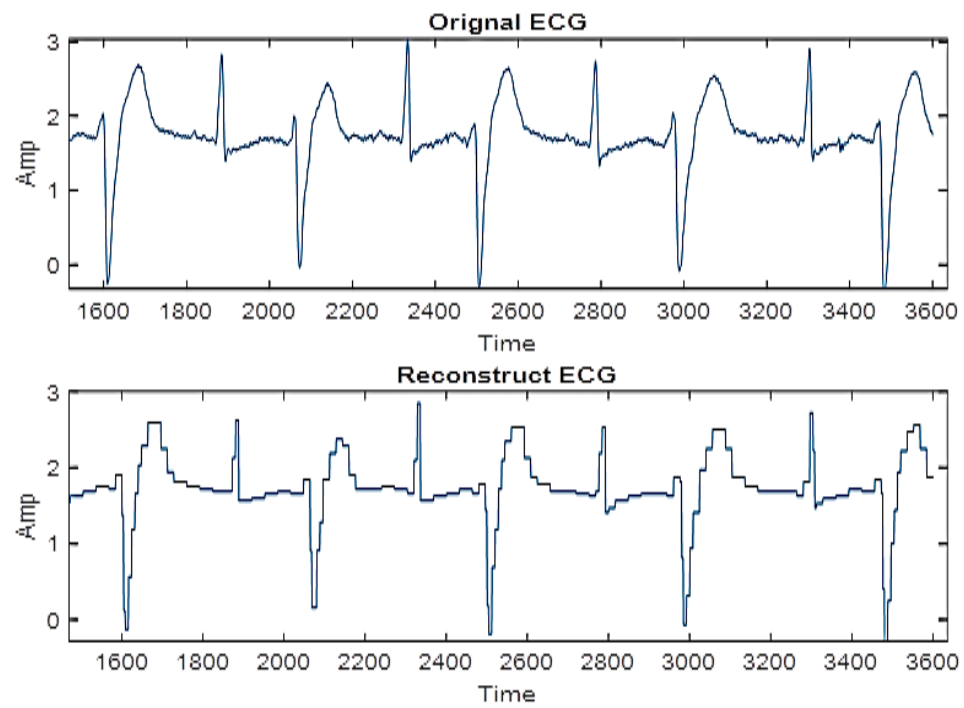

Figure 8. Original and reconstruct signal for record 200 with $C R=14.96 \& \mathrm{PRD}=5 \%$
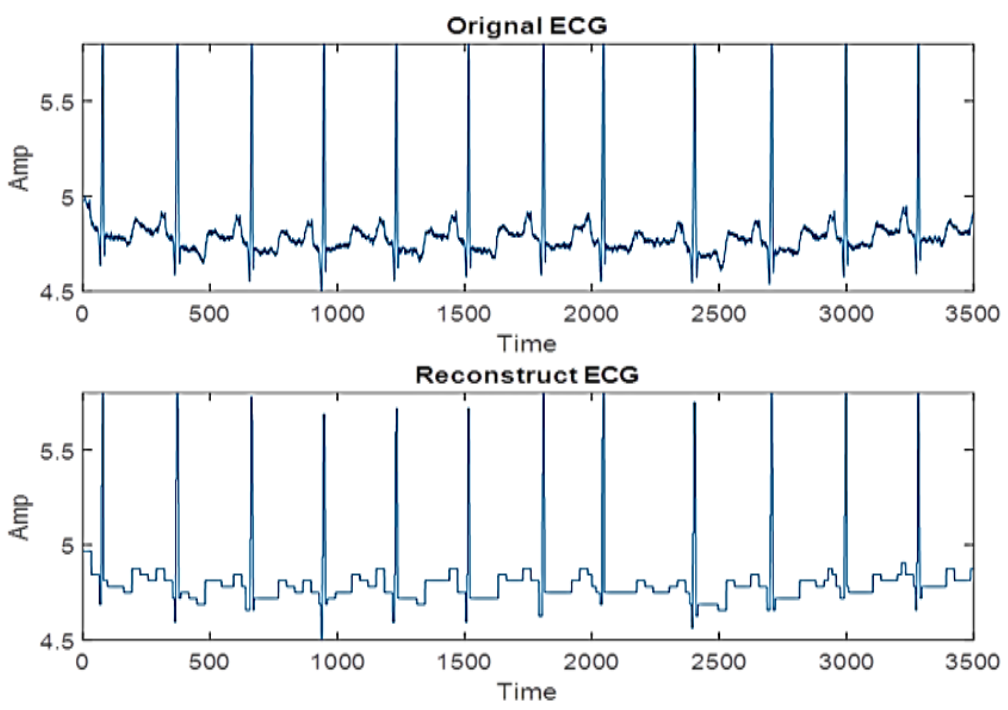

Figure 9. Original and reconstructed signal for $\mathrm{ECG}$ record 100 with $\mathrm{CR}=20.6$ and $\mathrm{PRD}=1 \%$

In order to compare comparison with another techniques, a suggested method was applied to records $100,109,115,119,200$. The wavelet decomposition up to 5 level by utilizing the wavelet Haar at quantization $(8 \mathrm{bit})$ is executed. The performance measures that used in order to evaluate the quality of the suggested algorithm are (CR and PRD). Tun et al. [23] presented an analysis, on ECG data compression using wavelet transform technique and reported a $\mathrm{PRD}=0.4275 \& \mathrm{CR}=10.7902$ for record 100 , while for record 109 achieved a $\mathrm{PRD}=0.1814 \& \mathrm{CR}=9.1709$. Chandra et al. [24] presented a comparative analysis of performance of several wavelet based ECG data compression methodologies and reported a PRD $=0.0765$ \& $\mathrm{CR}=13.1062$ for record 100 , while a record (109) achieved a $\mathrm{PRD}=0.0352, \mathrm{CR}=11.0609$. The suggested algorithm achieved percent root mean square error $(\mathrm{PRD})=1$ and compression ratio $(\mathrm{CR})=20.6$ when examined it on record 100 and achieved, while record 109 achieved PRD=5 and $\mathrm{CR}=15$. From the comparisons results a proposed algorithm is much superior when compared with other methods. The results of comparsion can be shown in Table 2 . 
Table 2. Introduce the comparison among a suggested method and another compression method based on wavelet

\begin{tabular}{lccc}
\multicolumn{4}{c}{ Wavelet } \\
\hline Algorithm & & PRD $(\%)$ & CR $(\%)$ \\
\hline Tun et.al [23] & 100 dat & 0.4275 & 10.7902 \\
& 109 dat & 0.1814 & 9.1709 \\
Chandra et.al [24] & 100 dat & 0.0765 & 13.1062 \\
& 109 dat & 0.0352 & 11.0609 \\
Jha and Kolekar [25] & 115 dat & 4.73 & 6.98 \\
& 119 dat & 10.83 & 8.15 \\
& 200 dat & 7.15 & 6.78 \\
Our algorithm & 100 & 1 & 20.6 \\
& 109 & 1 & 15 \\
& 115 & 5 & 18.11 \\
& 119 & 6 & 14.16 \\
& 200 & 5 & 14.96 \\
\hline
\end{tabular}

\section{CONCLUSION}

A 1-D signal compression is used through this work. A compression scheme that is utilized in a proposed technique is based on the discrete wavelet transform and dual encoding techniques (Huffman + RLE). This technique is tested on ECG signals that are taken from MIT-BIH database. A compression algorithm quality relys on a wavelet kind and the signal being compressed. Results show that the wanted quality description can be touched and compression is superior to another methods. After compression can observe whole clinical information is conserved and this making the suggested algorithm is secure for ECG signals compression.

\section{REFERENCES}

[1] K. S. Surekha and B. P. Patil, "Hybrid Technique For Ecg Signal Compression Using Parallel And Cascade Method," Biomedical Engineering: Applications, Basis and Communications, vol. 31, no. 1, 2019, doi: 10.4015/S1016237219500224.

[2] M. Brito, J. Henriques, P. Gil, and M. Antunes, "A predictive adaptive approach to generic ECG data compression," IEEE International Workshop on Intelligent Signal Processing, 2005., 2005, pp. 32-37, doi: 10.1109/WISP.2005.1531628.

[3] M. Abo-Zahhad and B. A. Rajoub, "An effective coding technique for the compression of one-dimensional signals using wavelet transforms," Medical Engineering \& Physics Journal, vol. 24, no. 3, pp. 185-199, 2002, doi: 10.1016/s1350-4533(02)00004-8.

[4] D. Biswas, E. B. Mazomenos, and K. Maharatna, "ECG compression for remote healthcare systems using selective thresholding based on energy compaction," 2012 International Symposium on Signals, Systems, and Electronics (ISSSE), 2012, pp. 1-6, doi: 10.1109/ISSSE.2012.6374306.

[5] M. Abo-Zahhad, A. F. Al-Ajlouni, S. M. Ahmed, and R. J. Schilling, "A new algorithm for the compression of ECG signals based on mother wavelet parameterization and best-threshold levels selection," Digital Signal Processing, vol. 23, no. 3, pp. 1002-1011, 2013, doi: 10.1016/j.dsp.2012.11.005.

[6] A. Al-Shrouf, M. Abo-Zahhad, and S. M. Ahmed, "A novel compression algorithm for electrocardiogram signals based on the linear prediction of the wavelet coefficients," Digital Signal Processing, vol. 13, no. 4, pp. 604-622, 2003, doi: 10.1016/S10512004(02)00031-3.

[7] M. L. Hilton, "Wavelet and wavelet packet compression of electrocardiograms," in IEEE Transactions on Biomedical Engineering, vol. 44, no. 5, pp. 394-402, May 1997, doi: 10.1109/10.568915.

[8] B. Bradie, "Wavelet packet-based compression of single lead ECG," in IEEE Transactions on Biomedical Engineering, vol. 43, no. 5, pp. 493-501, May 1996, doi: 10.1109/10.488797.

[9] K. Ranjeet, A. Kumar, and R. K. Pandey, "ECG Signal Compression Using Different Techniques," International Conference on Advances in Computing, Communication and Control, vol. 125, 2011, pp. 231-241, doi: 10.1007/978-3-642-18440-6 29.

[10] S. A. Chouakri, O. Djaafri, and A. Taleb-Ahmed, "Wavelet transform and Huffman coding based electrocardiogram compression algorithm: Application to telecardiology," Journal of Physics: Conference Series, 2013, doi: 10.1088/1742-6596/454/1/012086.

[11] M. M. Abo-Zahhad, A. I. Hussein, and A. M. Mohamed, "ECG Signal Compression Technique Based on Dwt and Exploitation of Interbeats And Intrabeats Correlations," Journal of Engineering Sciences, vol. 43, no. 6, pp. 837-856, 2015, doi: 10.21608/JESAUN.2015.115297.

[12] I. Daubechies, Ten Lectures on Wavelets (CBMS-NSF Regional Conference Series in Applied Mathematics), Pennsylvania, USA: SIAM: Society for Industrial and Applied Mathematics, 1992.

[13] M. Stéphane, A Wavelet Tour of Signal Processing The Sparse Way, Massachusetts, USA: Academic Press, 2009, doi: 10.1016/B9780-12-374370-1.X0001-8.

[14] M. Vetterli and C. Herley, "Wavelets and filter banks: theory and design," in IEEE Transactions on Signal Processing, vol. 40, no. 9, pp. 2207-2232, Sept. 1992, doi: 10.1109/78.157221.

[15] I. Daubechies, "Orthonormal bases of compactly supported wavelets," Communications on Pure and Applied Mathematics, vol. 41, no. 7, pp. 909-996, 1988, doi: 10.1002/cpa.3160410705.

[16] B. A. Rajoub, "An efficient coding algorithm for the compression of ECG signals using the wavelet transform," in IEEE Transactions on Biomedical Engineering, vol. 49, no. 4, pp. 355-362, April 2002, doi: 10.1109/10.991163.

[17] P. P. Vaidyanathan, Multirate Systems and Filter Banks, England, UK: Pearson College Div, 1992.

[18] Y. Zigel, A. Cohen, and A. Katz, "The weighted diagnostic distortion (WDD) measure for ECG signal compression," in IEEE Transactions on Biomedical Engineering, vol. 47, no. 11, pp. 1422-1430, Nov. 2000, doi: 10.1109/TBME.2000.880093.

[19] R. Benzid, F. Marir, and N-E. Bouguechal, "Quality-Controlled Compression Method using Wavelet Transform for Electrocardiogram Signals," International Journal of Medical and Health Sciences, vol. 1, no. 2, pp. 102-107, 2007, doi: 10.5281/zenodo.1085926. 
[20] S. Lloyd, "Least squares quantization in PCM," in IEEE Transactions on Information Theory, vol. 28, no. 2, pp. 129-137, March 1982, doi: 10.1109/TIT.1982.1056489.

[21] K. Sayood, Introduction to Data Compression, Massachusetts, USA: Morgan Kaufmann, 2016, doi: 10.1016/B978-0-12-6208627.X5000-7.

[22] S. Lee, J. Kim, and M. Lee, "A Real-Time ECG Data Compression and Transmission Algorithm for an e-Health Device," in IEEE Transactions on Biomedical Engineering, vol. 58, no. 9, pp. 2448-2455, Sept. 2011, doi: 10.1109/TBME.2011.2156794.

[23] H. M. Tun, W. K. Moe, and Z. M. Naing, "Analysis on ECG Data Compression Using Wavelet Transform Technique," International Journal of Psychological and Brain Sciences, vol. 2, no. 6, pp. 127-140, 2017, doi: 10.11648/j.ijpbs.20170206.12.

[24] S. Chandra, A. Sharma, and G. K. Singh, "A Comparative Analysis of Performance of Several Wavelet Based ECG Data Compression Methodologies," IRBM, vol. 42, no. 4, pp. 227-244, 2021, doi: 10.1016/j.irbm.2020.05.004.

[25] C. K. Jha and M. H. Kolekar, "Electrocardiogram data compression using DCT based discrete orthogonal Stockwell transform," Biomedical Signal Processing and Control, vol. 46, pp. 174-181, 2018, doi: 10.1016/j.bspc.2018.06.009.

\section{BIOGRAPHIES OF AUTHORS}

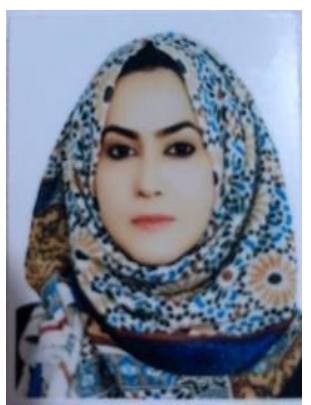

Rawaa Qasim Hamza (D) SC P was born in Baghdad, Iraq in 1990. She received his B.Sc degree in Electrical Engineering, Communication Engineering, University of Technology in 2013. Currently, she is studying a master's degree in Electronics and Communications Engineering in university of technology, Iraq. She can be contacted at email: 316314@student.uotechnology.edu.iq.

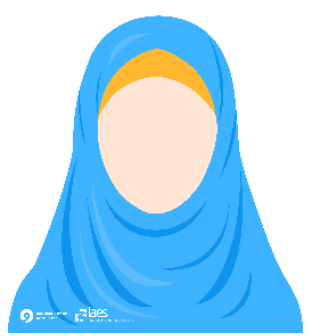

Khalida Shaaban Rijab (D) SC P was born in Baghdad, Iraq in 1964. She received his B.Sc degree in Control and Systems Engineering, University of Technology,Iraq in 1986, M.Sc. degree in Control Engineering, University of Technology, Iraq in 1994 and Ph.D. degree in Computer Engineering, University of Technology, Iraq in 2008, Since 2008, she works a lecturer at university of technology. She has many publications in national and international journals. She can be contacted at email: 30049@uotechnology.edu.iq.

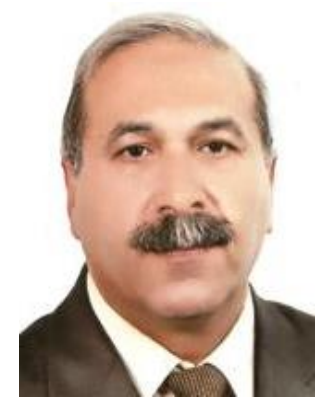

Mohammed Abdul Redha Hussien (iD SC P was born in Baghdad, Iraq in November of 1956. He received his B.Sc degree in Physics, College of Science, Baghdad University, Iraq in 1978 and M.Sc., Ph.D degree in Theoretical Physics, Baghdad University, Iraq in 1995, 2005 respectively. Since 2008, he works at university of technology. He has more 15 publications in national and international journals. His research interest in Optical Fiber, Solar cells, Thin Films, Theoretical Physics. He can be contacted at email: 14003@uotechnology.edu.iq. 